\title{
Particle Size Distribution Measurements of Manganese-Doped ZnS Nanoparticles
}

\author{
Yvonne Dieckmann, ${ }^{\dagger, \S}$ Helmut Cölfen, ${ }^{*}, *$ Heinrich Hofmann, ${ }^{\dagger}$ and Alke Petri-Fink*,† \\ Laboratory of Powder Technology, Ecole Polytechnique Fédérale de Lausanne, Lausanne, Switzerland, and Colloid \\ Chemistry Department, Max Planck Institute of Colloids and Interfaces, Research Campus Golm, Am Mühlenberg, \\ D-14424 Potsdam, Germany
}

We performed particle size and particle size distribution measurements for L-cysteine-stabilized $\mathrm{ZnS} / \mathrm{Mn}$ nanoparticles in the size region below $10 \mathrm{~nm}$. For this we applied transmission electron microscopy (TEM), analytical ultracentrifugation (AUC), dynamic light scattering (DLS), and asymmetric flow field flow fractionation (aF-FFF) measurements, and we calculated particle sizes with the help of X-ray diffraction (XRD) patterns and the shift of the band gap absorption in the UV-vis spectrum. The different methods are explained, and their limitations are discussed, with the conclusion that only a combination of different techniques can yield a realistic and complete picture about the size distribution of the sample. From these methods TEM, AUC, DLS, and aF-FFF measure the actual particle size distribution either in dispersion or after drying of the sample, whereas the particle size obtained from XRD patterns and with the help of the band gap widening corresponds to the average size of the crystal domains within the particles. We obtained particle size distributions with their maximum between 3 and $7 \mathrm{~nm}$ and a mean crystallite size of 3.5-4 $\mathrm{nm}$.

The synthesis of nanometer-sized colloidal particles has been intensively studied in recent years because of their considerable technological and fundamental scientific interest. This arises from the remarkable variations in fundamental physical properties that occur when one changes from a macroscopic solid to a particle consisting of a countable number of atoms. ${ }^{1-3}$ Semiconductor nanoparticles have attracted profound interest in the past. Variation of fundamental characteristics ranging from phase transitions to electrical conductivity can be induced by controlling the size of these crystals. ${ }^{4,5}$ These changes arise from a spatial confinement of the exciton when the particle reaches the size of the de Broglie

\footnotetext{
* To whom correspondence should be addressed. Phone: +49 331 567-9513 (H.C.); +41 (0) 216935107 (A.P.-F.). Fax: +49 331 567-9502 (H.C.); +41 (021) 6933089 (A.P.-F.). E-mail: Coelfen@mpikg.mpg.de (H.C.); alke.fink@ epfl.ch (A.P.-F.).

${ }^{\dagger}$ Ecole Polytechnique Fédérale de Lausanne.

* Max Planck Institute of Colloids and Interfaces.

$\S$ Current address: Research Solution Polymers, BASF Aktiengesellschaft, 67056 Ludwigshafen, Germany.

(1) Schmid, G. Nanoparticles: From Theory to Application; Wiley VCH: Weinheim, Germany, 2004.

(2) Schmid, G. Clusters and Colloids; VCH Press: New York, 1994.

(3) Moriarty, P. Rep. Prog. Phys. 2001, 64, 297.

(4) Alivisatos, A. P. J. Phys. Chem. 1996, 100, 13226.

(5) Eychmüller, A. J. Phys. Chem. B 2000, 104, 6514.
}

wavelength. This leads to a widening of the band gap with decreasing particle size. ${ }^{6}$ Applications are found in the field of medical and biological sensors where such particles represent a promising alternative to commonly used organic dyes, due to their change of emission color with particle size. ${ }^{7,8}$ Since these changes depend on the size of the system, size characterization techniques are necessary to predict the degree of variation in physical properties. ${ }^{9}$ However, most reports on semiconductor nanoparticles deal with the optical properties of these crystals and do not pay attention to particle size (distributions) apart from transmission electron microscopy images and crystallite sizes derived from $\mathrm{X}$-ray diffraction patterns.

There are a vast number of methods for particle size analysis, and the detailed description and comparison between them is clearly beyond the scope of this work. However, the used techniques will briefly be described in the next section.

In this work, we have applied a range of analytical techniques for the determination of particle sizes to investigate the information content and limitations of the different measurements. A similar study was conducted several years ago in the company Bayer for various latices and latex mixtures in the size range of $80-1500$ $\mathrm{nm} .{ }^{10}$ For these samples, of the methods to determine only average particle sizes, turbidimetry proved to be the most efficient, followed by dynamic light scattering (DLS) with cumulants evaluation. Cumulants fits should only be applied to samples with a polydispersity $<10 \%$ and are of limited use for polydisperse samples. ${ }^{11}$ Static light scattering (SLS) only yielded accurate results for small particles with narrow particle size distributions.

For the determination of particle size distributions, analytical ultracentrifugation (AUC) and, somewhat less, disk centrifugation and electron microscopy with image analysis proved to be the most efficient. However, for very small inorganic nanoparticles the situation may be different. A similar study on interacting latexbased systems revealed AUC as the most accurate method for the determination of the particle sizes and quantities in a mixture, transmission electron microscopy gave visual information, and SLS

(6) Gaponenko, S. V. Optical Properties of Semiconductor Nanocrystals; Cambridge University Press: Cambridge, 1998.

(7) Parak, W. J.; Gerion, D.; Pellegrino, T.; Zanchet, D.; Micheel, C.; Williams, S. C.; Boudreau, R.; Le Gros, M. A.; Larabell, C. A.; Alivisatos, A. P. Nanotechnology 2003, 14, R15.

(8) Bruchez, M., Jr.; Moronne, M.; Gin, P.; Weiss, S.; Alivisatos, A. P. Science 1998, 281, 2013.

(9) Allen, T. Particle Size Measurements; Chapman and Hall: London, 1990.

(10) Lange, H. Part. Part. Syst. Charact. 1995, 12, 148.

(11) Hassan, P. A.; Kulshreshtha, S. K. J. Colloid Interface Sci. 2006, 300/2, 744 .

Analytical Chemistry, Vol. 81, No. 10, May 15, 2009

3889 
was the most suitable technique to study aggregation kinetics. ${ }^{12}$ A similar result was obtained for the comparison of AUC and DLS for growing $\mathrm{ZrO}_{2}$ colloids. ${ }^{13}$ Very recently, a comparison of sedimentation velocity ultracentrifugation with DLS and transmission electron microscopy was published for the analysis of small $\left(d_{\mathrm{H}}\right.$ ca. $\left.15 \mathrm{~nm}\right)$ silica nanoparticles showing that AUC yielded the most reliable particle size information. ${ }^{14}$ In our study, we intended to extend the range of compared techniques and investigate an even smaller sample: a manganese-doped $\mathrm{ZnS}$ semiconductor.

\section{MATERIALS AND METHODS}

Manganese-doped $\mathrm{ZnS}$ nanoparticles were synthesized by coprecipitation of $\mathrm{Zn}^{2+}$ and $\mathrm{Mn}^{2+}$ precursors using $\mathrm{Na}_{2} \mathrm{~S}$ in the presence of L-cysteine as stabilizer. ${ }^{15,16}$ After dialysis, transparent dispersions were obtained, and the powders were isolated by freeze-drying after dialysis. The thus obtained powders and dispersions were further investigated by transmission electron microscopy (TEM), X-ray diffraction (XRD), photon correlation spectroscopy (DLS), asymmetric flow field flow fractionation (aFFFF), AUC, and UV-vis absorption spectroscopy. Solvent was water.

Transmission electron microscopy was performed on a Phillips CM-20 microscope operating at $200 \mathrm{kV}$. For sample preparation, diluted drops of suspensions were allowed to dry slowly on carboncoated copper grids. Microscopy has the obvious advantage that information about both morphology and size distribution can be obtained at the same time. The major disadvantage is that the method is problematic in case of polydispersity due to limited statistics for detected particles and possible drying artifacts like aggregation or sample fractionation.

Dynamic light scattering is based on the light scattered by (colloidal) particles which are undergoing random thermal motions. The scattered light fluctuations can be related the diffusion coefficient of a particle, which in turn can be converted to a particle diameter. For sub-200 nm narrow size particles, DLS is a very good and fast method. Main problems arise for large size distributions and/or multimodal distributions. DLS is capable of measuring down to 1-2 $\mathrm{nm}$; however, a high-intensity laser and the exclusion of dust are imperative. Light scattering measurements were carried out at $90^{\circ}$ on a photon correlation spectrometer (DLS) from Brookhaven equipped with a BI-9000AT digital autocorrelator. The CONTIN method was used for data processing but with care to modify data collection parameters and baseline choices for the finer particles investigated below $50 \mathrm{~nm}$. All the information is coded in the correlation function, which is then mathematically treated to give a size distribution. As such handling is complex, and no exact method exists for the correlation function fitting, many approaches can be used. The main drawback or difficulty is that any correlation function can be approximated by different models with the same accuracy. Among the classical approaches some primitive processes (which

(12) Cölfen, H.; Völkel, A.; Eda, S.; Kobold, U.; Kaufmann, J.; Puhlmann, A.; Göltner, C.; Wachernig, H. Langmuir 2002, 18, 7623.

(13) Cölfen, H.; Schnablegger, H.; Fischer, A.; Jentoft, F. C.; Weinberg, G.; Schlögl, R. Langmuir 2002, 18, 3500.

(14) Planken, K. L.; Kuipers, B. W. M.; Philipse, A. P. Anal. Chem. 2008, 80, 8871-8879.

(15) Axmann, Y.; Petri, A.; Hofmann, H. Mater. Res. Soc. Symp. Proc. 2004, 789, N11.23.

(16) Bae, W.; Mehra, R. K. J. Inorg. Biochem. 1998, 70, 125. can be applied for "simple" size distributions investigation) are usually available as well as the so-called CONTIN method for more complex size distributions (allowing multimodal distribution analysis). In this case, the intensity-weighted radius distribution is obtained by direct numerical inversion of the DLS data. ${ }^{17}$ The limit of this method mainly consists in the numerical difficulty of the inversion procedure for a finite number of experimental data. However, the CONTIN method was chosen because it always showed the best fit for the correlation function and gave reproducible results. Moreover, it is a well-known and widely used method for PCS data processing facilitating comparison with other studies. The water used for dispersion medium preparation was always filtered on $20 \mathrm{~nm}$ ceramic filters (Whatman, Anodisc, U.K.). Viscosity and refractive index of pure water were used for PCS size distribution calculation.

X-ray diffraction patterns were obtained with a Siemens Kristalloflex 805 equipped with a copper source (instrument broadening of $0.06 \mathrm{rad}$ ). All samples were analyzed under the same conditions (continuous mode, step size of $0.02^{\circ}$, and step time of $3 \mathrm{~s})$. The data were processed using the Powdercell software. When crystallites are smaller than approximately $100 \mathrm{~nm}$ in size, appreciable broadening in the XRD lines will occur. These regions may in fact correspond to the actual size of the particles. At other times, however, these regions form "domains" in the larger particle and may be a distinguishing and important feature. Therefore, particle size and crystallite size do not have the same meaning. Particles can be composed from several or many small crystallites. In either case, the observed line broadening can be used to estimate the average size. In the simplest case where the particles are stress-free, the size is estimated from a single diffraction peak. The thus obtained average crystallite size can help support observations made using the other above-mentioned techniques.

Analytical ultracentrifugation is a fractionating absolute technique for determination of molar mass and particle size (distributions) with high statistical relevance as every particle is detected. ${ }^{18}$ An important parameter for the evaluation is the knowledge of the partial specific volume, respectively, the density of the sample, which can be problematic for charged, small, and hybrid nanoparticles. Analytical ultracentrifugation was performed on an Optima XL-I (Beckman Coulter, Palo Alto, CA) using the absorption optics at a detection wavelength of $270 \mathrm{~nm}$ because of the highest absorbance around this wavelength and speeds of $10000-40000 \mathrm{rpm}$ at $25^{\circ} \mathrm{C}$ for all samples. Different speeds were necessary because of the strongly varying sedimentation coefficients. The non-diffusion-corrected sedimentation coefficient distribution ls- $g(s)$ was calculated using the program SEDFIT. ${ }^{19}$ The particle size distribution was calculated according to

$$
d_{\mathrm{H}}=\sqrt{\frac{18 \eta s}{\rho_{\mathrm{P}}-\rho}}
$$

with $d_{\mathrm{H}}$ being the hydrodynamic diameter, $\eta$ the solvent viscosity, $\rho_{\mathrm{P}}$ the particle density, $\rho$ the solvent density, and $s$ the sedimentation coefficient assuming compact spherical

(17) Finsy, R.; De Jaeger, N.; Sneyers, R.; Geladé, E. Part. Part. Syst. Charact. 1992, 9, 125-137.

(18) Mächtle, W.; Börger, L. Analytical Ultracentrifugation of Polymers and Nanoparticles; Barth, H. G., Pasch, H., Eds.; Springer Laboratory Manuals in Polymer Science; Springer Verlag: Berlin and Heidelberg, 2006.

(19) Schuck, P. Biophys. J. 2000, 78, 1606. 

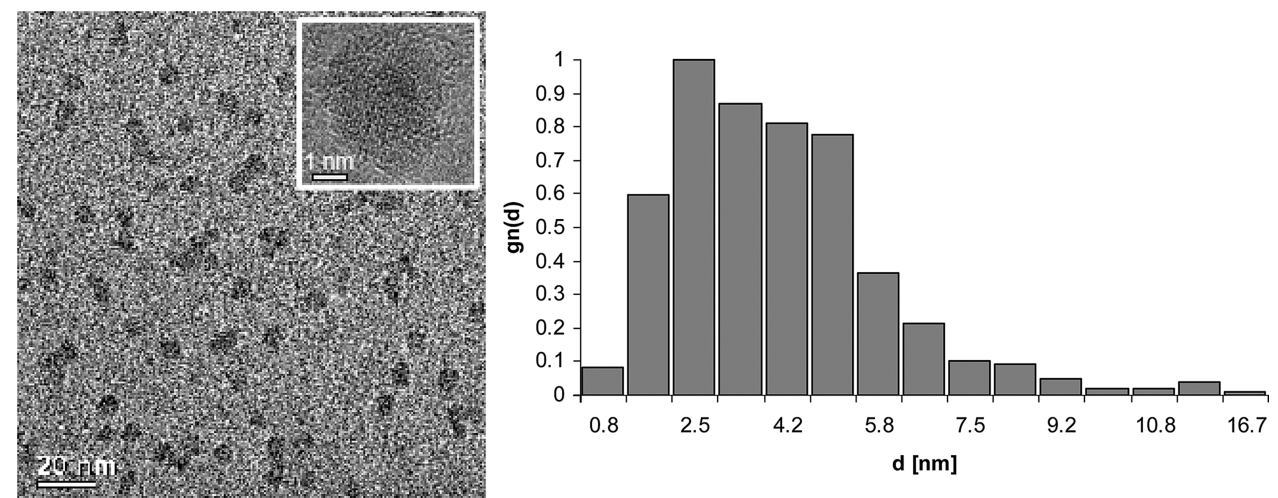

Figure 1. Left: TEM micrograph of $\mathrm{ZnS} / \mathrm{Mn}$ nanoparticles. Inset: representative high-resolution electron micrograph of one $\mathrm{ZnS} / \mathrm{Mn}$ nanoparticle (aspect ratio 1.1). Right: typical number-weighted particle size distribution $g_{n}(d)$ of $\mathrm{ZnS} / \mathrm{Mn}$ particles obtained by counting 540 nanoparticles from transmission electron micrographs.

particles. This equation assumes a hard sphere at infinite dilution, and therefore, it is assumed that the particle diameter equals the hydrodynamic diameter. In case of solvation, or a stabilizer shell, the density will be lower than that of the bulk material and the hydrodynamic particle diameter will be bigger than that of the particle core.

Field flow fractionation is a family of analytical separation techniques for polymers and particles employing a variety of physical fields for the separation of the sample. ${ }^{20} \mathrm{FFF}$ can be performed with a standard high-performance liquid chromatography (HPLC) setup by replacing the HPLC column with the FFF channel. Among the different FFF techniques, aF-FFF is the most popular. The applied cross-flow field allows for the absolute determination of a diffusion coefficient distribution. aF-FFF was performed at $25^{\circ} \mathrm{C}$ on an asymmetric channel (Consenxus, Ober Hilbersheim) with a spacer of $0.0320 \mathrm{~cm}$ thickness and a regenerated cellulose membrane (Postnova, Munich) having a molecular weight cutoff of $5000 \mathrm{~g} / \mathrm{mol}$. The carrier liquid was delivered by a Consenxus pump P 1.0 and degassed by a Degasys (Japan) DG-1310 using water as an eluent. The eluent flow was $0.5 \mathrm{~mL} / \mathrm{min}$, and the cross-flow was $2 \mathrm{~mL} / \mathrm{min}$. The sample was injected by a $10 \mathrm{~mL}$ Knauer (Berlin) injection pump, and the flow conditions were controlled by a flowbox and controller from Consenxus and a Bronkhorst Liqui-Flow control valve. As a detector, a Bischoff (Leonberg) Lambda 1000 UV-vis detector was applied at $280 \mathrm{~nm}$. The diffusion coefficient distributions from ${ }^{a F-F F F}{ }^{20}$ were converted to the particle size distributions by means of the Stokes-Einstein equation, which assumes hard spheres at infinite dilution so that the hydrodynamically equivalent sphere is calculated: ${ }^{21}$

$$
d_{\mathrm{H}}=\frac{k T}{3 \pi \eta D}
$$

with $k$ being Boltzmann's constant, $T$ the absolute temperature, $d_{\mathrm{H}}$ the hydrodynamic particle diameter, $D$ the translational diffusion coefficient, and $\eta$ the solvent viscosity.

UV-vis spectroscopy was performed on a Perkin-Elmer Lambda 900 spectrometer. The shape of the absorption spectrum in the vicinity of the absorption edge for a bulk semiconductor is usually determined by the nature of the electronic transition from the valence band to the conduction band. When the size of the system becomes smaller than the exciton radius, quantum confinement leads to size-dependent enlargement of the band gap and results in a blue-shift in the absorption onset. The relationship between band gap and size of semiconductor nanoparticle can be obtained using a number of models. ${ }^{22}$ Here we used the effective mass model for spherical particles with a Coulomb interaction term. ${ }^{23}$ This approach fails for the smallest crystallite sizes because of the oversimplified description of the crystal potential as a spherical well with an infinitely high potential at the interface. ${ }^{24}$

The different applied techniques are sensitive to different particle diameters. AUC, DLS, and aF-FFF yield the hydrodynamic diameter $d_{\mathrm{H}}$, which includes a solvation or stabilizer shell if applicable. In case of the here investigated cystein-stabilized $\mathrm{ZnS}$ nanoparticles, a stabilizer shell of up to $0.6 \mathrm{~nm}$ thickness can be assumed as found for the length of an attached cystein molecule by molecular modeling (not shown). On the other hand, TEM and XRD deliver the diameter $d$ of the inorganic particle core since the organic shell is less electron dense compared to the inorganic core and thus transparent in TEM and XRD.

\section{RESULTS AND DISCUSSION}

Transmission electron microscopy allows particle size measurement of dispersions with narrow size distributions. To obtain a particle size distribution from transmission electron micrographs we manually measured particle sizes for 540 particles to ensure a reliable representation of the actual size distribution. ${ }^{25} \mathrm{~A}$ typical TEM image and the obtained particle size distribution can be seen in Figure 1.

The major challenge lies in the distinction between single particles and agglomerates consisting of two or three single particles. In addition, the images do not necessarily represent the in situ situation in dispersion, since agglomerate formation might be due to sample preparation.

The obtained number-weighted particle size distribution ranges from 2 to $11 \mathrm{~nm}$ with either larger particles or agglomerates up to $17 \mathrm{~nm}$ and is shown in Figure 1. The larger sizes are attributed to the particles that were counted as single particles when no

(20) Cölfen, H.; Antonietti, M. Adv. Polym. Sci. 2000, 150, 67.

(21) Stokes, G. G. Trans. Cambridge Philos. Soc. 1850, 9, 8-106.

(22) Efros, A. L.; Rosen, M. Annu. Rev. Mater. Res. 2000, 30, 475.

(23) Brus, L.E. J. Chem. Phys. 1984, 80, 4403.

(24) Lippens, P. E.; Lannoo, M. Phys. Rev. B 1989, 39, 109359.

(25) Delannay, F., Ed. Characterization of Heterogeneous Catalysts; Marcel Dekker, Inc.: Basel, Switzerland, 1984. 


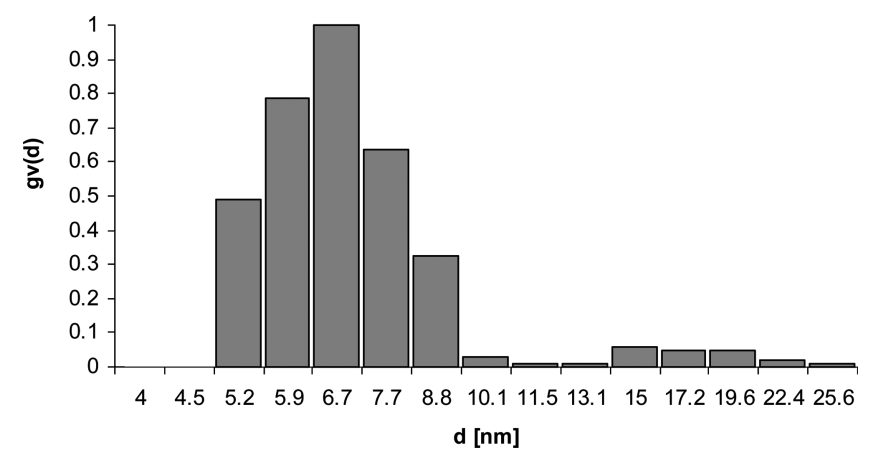

Figure 2. Typical volume-weighted particle size distribution $g_{\mathrm{v}}(d)$ of $\mathrm{ZnS} / \mathrm{Mn}$ particles obtained by photon correlation spectroscopy.

distinction between single particle and agglomerate was possible from the micrograph.

Particle sizes from XRD patterns were estimated using Scherrer's equation. This method uses the full peak width at halfmaximum (fwhm) and thus avoids the determination of the full peak profile. On the basis of the assumption that no strain contributed to the profile, the following relation between the fwhm $\beta_{1 / 2}$, the X-ray wavelength $\lambda$, and the crystallite size $d$ is applied, where $\Theta$ represents the Bragg angle: ${ }^{26}$

$$
\beta_{12}=\frac{0.94 \lambda}{d \cos \Theta}
$$

From this, a mean crystal sizes of $3.6 \pm 0.4 \mathrm{~nm}$ was obtained (the error being determined from the standard deviation over the main peaks). It must be emphasized that the size determined by diffraction methods corresponds to the magnitude of the coherent crystal regions, that is, to regions where the periodic arrangement of the atoms is perfect and continuous. Therefore, the size obtained by diffraction cannot always be simply compared to the sizes determined by other techniques.

Photon correlation spectroscopy or dynamic light scattering is a method which depends on the interaction of light with particles. ${ }^{27}$ This method is especially well suited for the measurement of narrow particle size distributions in the range of $2-500$ $\mathrm{nm}$ as polydispersity can distort the results due to the lack of sample fractionation. DLS is capable of measuring particle sizes down to $2-3 \mathrm{~nm}$, but a high-intensity laser and the preclusion of contaminations and dust is required. Figure 2 shows a volumeweighted particle size distribution measured by DLS. In order to allow detection of very small particles the dispersion had to be centrifuged at $5000 \mathrm{rpm}$ for $15 \mathrm{~min}$ to eliminate dust.

The main peak of the DLS distribution is found between 5 and $10 \mathrm{~nm}$ with a maximum at $7 \mathrm{~nm}$, but nevertheless, particles up to $25 \mathrm{~nm}$ were detected. It cannot be ruled out that centrifugation of the sample eliminates larger particles and/or agglomerates from the dispersion in addition to dust. However, calculations for particles up to $100 \mathrm{~nm}$ show that they do not fully sediment in the centrifuge under the chosen conditions.

Another technique to determine sizes of polymers, biomolecules, and colloidal dispersions is FFF. ${ }^{20} \mathrm{FFF}$ is a family of

(26) Lu, S. W.; Lee, B. I.; Wang, Z. L.; Tong, W.; Wagner, B. K.; Park, W.; Summers, C. J. J. Lumin. 2001, 92, 73.

(27) Chu, B. Laser Light Scattering: Basic Principles and Practice, 2nd ed.; Academic Press: New York, 1992.

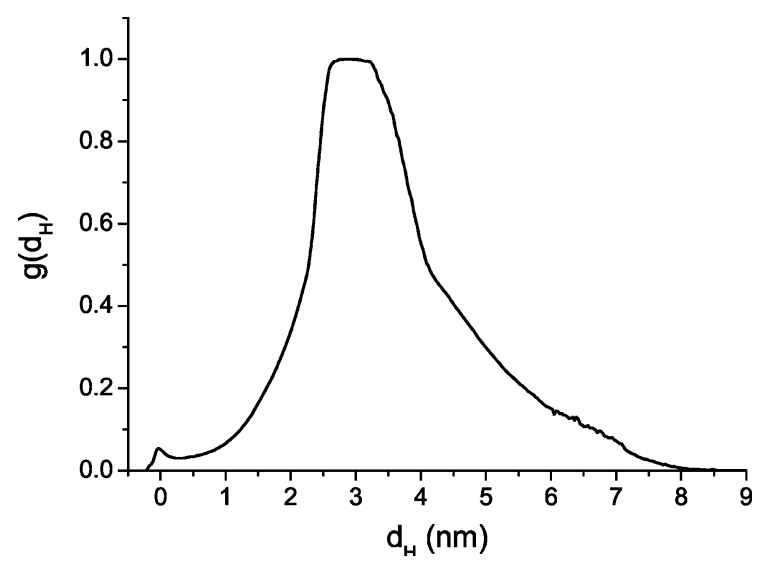

Figure 3. Typical mass-weighted particle size distribution $g_{w}\left(d_{H}\right)$ obtained with asymmetrical aF-FFF and UV-vis detection.

chromatographic techniques which have the advantage that the separation is achieved solely through the interaction of the sample with an external physical field and without a stationary phase. For the determination of absolute particle size distributions flow field flow fractionation (F-FFF) is useful-especially the here applied asymmetrical aF-FFF. ${ }^{16}$ Due to the applied UV-vis detector, which is mass-sensitive, the obtained distributions are massweighted. From this, the volume-weighted distributions can be determined by division through the particle density, if a constant particle density can be assumed. The resulting mass distribution for our particle dispersions is shown in Figure 3.

The obtained size distribution has its maximum at $3.5 \mathrm{~nm}$, which is considerably smaller than what was obtained by DLS and TEM after suitable conversion to a mass-weighted distribution (see Figure $5 \mathrm{~b}$ for comparison of the mass-weighted distributions). This can be explained by the surface charge of the nanoparticles. Since the particles are equally charged they repel one another. This is unproblematic when working at low concentrations, but when the sample is pressed to the accumulation wall by the crossflow, a locally high concentration is created, and the repulsion of the particles adds to the back diffusion of the particles to the center of the channel resulting in earlier elution and apparently smaller particle sizes. To prevent this, sodium chloride to screen the surface charges and Tween to prevent agglomeration are normally added to measure charged species. Unfortunately, the particle dispersions are very sensitive to changes in ionic strength, and Tween could not prevent agglomeration. Thus, we measured our particles in pure water, and the particle size distributions obtained with these measurements are probably shifted to smaller sizes compared with the actual value for particle size.

Another hydrodynamic method to determine particle size distributions-especially of ultrafine nanoparticles and quantum dots-is AUC. ${ }^{28} \mathrm{AUC}$ is a fractionation technique where separation due to particle size and density difference of particles and solvent takes place in a centrifugal field. A UV-vis or refractive index detector observes the migration in the centrifugal field which leads to mass-weighted sedimentation coefficient distributions. ${ }^{28}$ Since the measured dispersions do not consist of pure $\mathrm{ZnS}$ particles dispersed in water, but of cysteine-stabilized particles, the bulk

(28) Cölfen, H.; Pauck, T. Colloid Polym. Sci. 1997, 275, 175. 


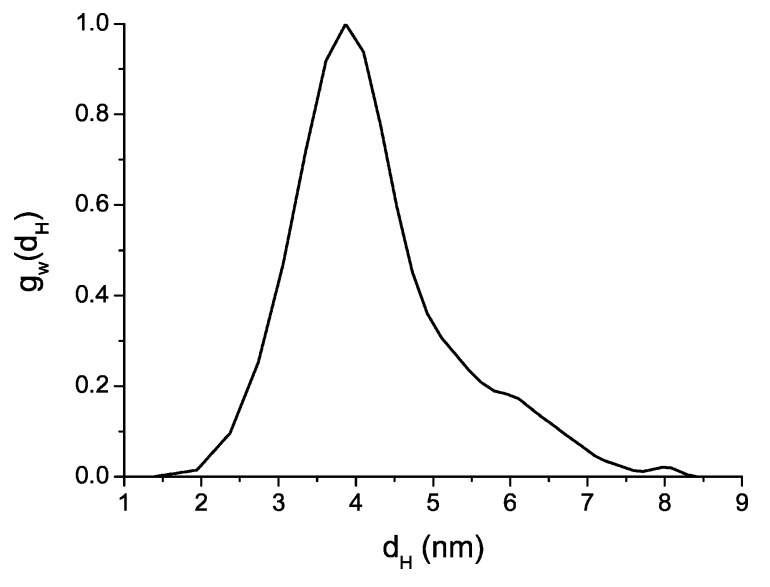

Figure 4. Mass-weighted particle size distribution $g_{w}\left(d_{H}\right)$ for AUC measurements measured at $350 \mathrm{nmol} / \mathrm{L}$, calculated with a density of $3.2 \mathrm{~g} / \mathrm{mL}$.

ZnS density of $4.1 \mathrm{~g} / \mathrm{mL}$ cannot be used. ${ }^{29}$ Helium pycnometry was used to estimate the actual density of $3.2 \mathrm{~g} / \mathrm{mL}$ since the specific volume of our core-shell sample could not be determined from the same sedimentation velocity experiment by the procedure described by Planken et al. ${ }^{14}$ In analogy to FFF, surface charges can lead to particle repulsion at high concentrations and therefore to a higher back diffusion relevant for aF-FFF and slower sedimentation relevant for AUC. From this arises the custom for AUC measurements to extrapolate sedimentation coefficients, measured at finite concentration, to infinite dilution. This concentration dependence is very strong as shown in Supporting Information Figure SI 1, although the investigated concentrations are very small in the micromolar range as result of the high extinction coefficient of the material. In Supporting Information Figure SI 1a, significant sharpening of the sedimentation coefficient distribution with increasing sample concentration can be detected due to particle charge, while the concentration dependence of the inverse sedimentation coefficient in Supporting Information Figure SI $1 \mathrm{~b}$ shows two regimes.

Above ca. $2.5 \mu \mathrm{mol} / \mathrm{L}$, the inverse sedimentation coefficient does not change much anymore as the particles strongly repel each other in this range. Below $2.5 \mu \mathrm{mol} / \mathrm{L}$, the expected linear dependence yields a sedimentation coefficient of $33 \mathrm{~S}$ for infinite dilution. This is significantly higher than the one of $20.6 \mathrm{~S}$ determined for the most diluted solution of $350 \mathrm{nmol} / \mathrm{L}$. However, due to detection limits, we could not carry out experiments for still lower concentrations so that the particle size distribution from AUC underestimates the particle size. Figure 4 shows the particle size distribution obtained from a sedimentation coefficient distribution assuming a particle density in dispersion of $3.2 \mathrm{~g} / \mathrm{mL}$.

The distribution has a maximum at around $4 \pm 1 \mathrm{~nm}$ and a tail at larger particle sizes. The error corresponds to density inaccuracy. Since the particles are, in addition to the cysteine-adsorbed layer, hydrated in dispersion it is probable that the density is even smaller than $3.2 \mathrm{~g} / \mathrm{mL}$. This would shift the distribution to larger particle sizes. The shift of the maximum of the size distribution from 4 to $5 \mathrm{~nm}$ requires a density of $2.3 \mathrm{~g} / \mathrm{mL}$. Therefore, even with a very small density the obtained data still is in the size ranges determined by DLS and TEM.

(29) CRC Handbook of Chemistry and Physics; Weast, R. C., Ed.; CRC Press Inc.: Boca Raton, FL, 1989.
Since the size-related band gap shift of semiconductor nanocrystals can be quantified, it is possible to calculate an optical particle size with the band gap shift measured from absorption spectra. ${ }^{30}$ For this, the effective mass approximation is used:

$$
\begin{aligned}
E=E_{\mathrm{g}}+\frac{\pi^{2} \hbar}{2 m_{0}{ }^{2}}\left(\frac{1}{m_{\mathrm{e}}^{\star}}+\frac{1}{m_{\mathrm{h}}^{\star}}\right)-\frac{1.8 e^{2}}{4 \pi \varepsilon_{0} \varepsilon_{\mathrm{r}} r}-0.25 \frac{e^{4}}{8 \pi^{2} \varepsilon_{0}{ }^{2} \varepsilon_{\mathrm{r}}^{2} \hbar} \times \\
\left(\frac{1}{m_{\mathrm{e}}^{\star}}+\frac{1}{m_{\mathrm{h}}^{\star}}\right)^{-1}
\end{aligned}
$$

where $m_{\mathrm{e}}^{\star}$ and $m_{\mathrm{h}}^{\star}$ are the effective masses of the electron and the hole, $r$ is the radius of the particle, $\varepsilon_{0}$ is the permittivity of the vacuum, $\varepsilon_{\mathrm{r}}$ is the relative permittivity of the particle, and $E_{\mathrm{g}}$ is the bulk band gap ( $3.6 \mathrm{eV}$ for $\left.\mathrm{ZnS}\right)$. With effective masses of $m_{\mathrm{e}}=0.25$ and $m_{\mathrm{h}}=0.59\left(m_{\mathrm{e}}^{*}=m_{\mathrm{e}} / h m_{0}\right), \varepsilon_{\mathrm{r}}=8.5$, and an onset of absorption of $310 \mathrm{~nm}$ (data not shown), a particle size of $4 \pm 0.4 \mathrm{~nm}$ is obtained as number average, since two UV-vis spectra of nanoparticles of different size average to that which would correspond to the number-average particle size $(\mathrm{H}$. Cölfen, unpublished observations). The error corresponds to $\pm 10 \mathrm{~nm}$ for the determination of the onset of absorption.

This approach fails for the smallest $(r \leq 1 \mathrm{~nm})$ particles because of the oversimplified description of the crystal potential as a spherical well with an infinitely high potential at the interface. In addition, the effective masses have been assumed to be constant which is only correct for electronic states near the band edge. ${ }^{31}$ It was shown in another study that the effective masses for $\mathrm{PbS}$ actually increased with decreasing particle size. ${ }^{32}$

Table 1 summarizes the results obtained from the different analytical methods. For a better comparison, the number-weighted particle size distribution was transformed into the mass-weighted particle size distribution by multiplication of the counts for each particle size with the respective particle size and normalization. Likewise, the volume distribution was converted into the mass distribution by multiplication of the respective frequencies with the particle density of $3.2 \mathrm{~g} / \mathrm{mL}$. Thus, the volume-weighted distribution was equal to the mass distribution after renormalization. It has to be noted that it is always important to consider how the obtained particle size (distribution) is weighted as differences in the particle size will result from different weighing.

The analytical methods can be separated in two groups: the TEM, AUC, DLS, and aF-FFF measurements that provide particle size distributions and XRD and absorption spectroscopy that result in a mass- and number-average particle size, respectively. The maxima of the modes of the particle size distributions in Table 1 show the maxima for DLS, TEM, and AUC measurements between 5 and $8 \mathrm{~nm}$, whereas the maximum for the aF-FFF measurements is at $3.5 \mathrm{~nm}$. For aF-FFF it was already discussed that the smaller particle size compared to TEM and AUC is probably due to the negative charges of the particles that lead to an additional force for back diffusion to the channel center and thus earlier elution than corresponding to the particle size. This results in too low particle sizes. DLS on the other hand suffers from particle aggregates and polydispersity-the determined

(30) Brus, L. E. J. Chem. Phys. 1983, 79 (11), 5566.

(31) Henglein, A. Chem. Rev. 1989, 89, 1861.

(32) Wang, Y.; Heron, N.; Moller, K.; Bein, T. Solid State Commun. 1991, 77, 33. 

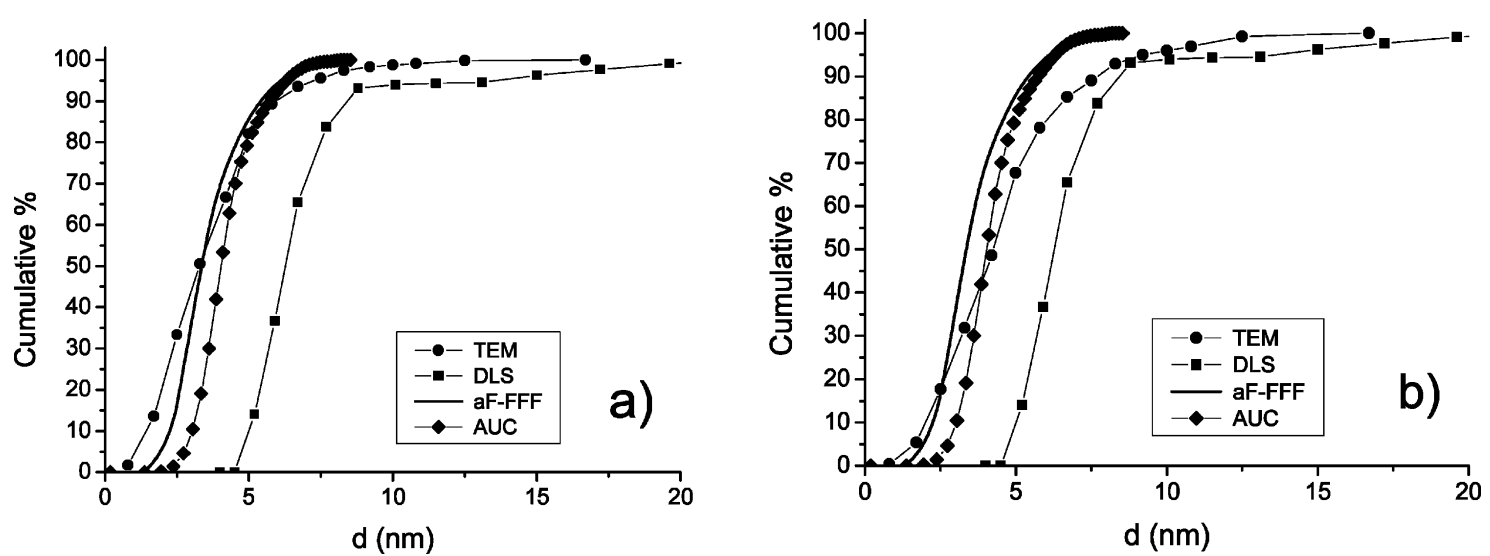

Figure 5. Cumulative representations of AUC, TEM, and DLS particle size distributions. The left figure shows the distributions as obtained from the different analytical techniques. The right figure shows all distributions weighted by mass.

\section{Table 1. Average Particle Size Obtained from AUC, DLS, aF-FFF, XRD, TEM, and Absorption Measurements ${ }^{a}$}

weighed size $(\mathrm{nm})$, type of average average mass-

AUC

DLS

$\mathrm{XRD}$

TEM

$4.5 \pm 1.1$ (mass) $4.9 \mathrm{~nm}$ (mass)*

$7.4 \pm 2.9$ (volume)

$3.5 \pm 1.0$ (mass)

$3.6 \pm 0.4$ (mass)

$4.0 \pm 2.0$ (number)
$4.0 \pm 0.5$ (number)

$4.5 \pm 1.1 ; 4.9 \mathrm{~nm}^{*}$

$7.4 \pm 2.9$

$3.5 \pm 1.0$

$3.6 \pm 0.4$

$5.0 \pm 2.5$

N.A. weighted size $[\mathrm{nm}]$

aF-FFF

${ }^{a}$ For AUC, the average size from extrapolation of the average sedimentation coefficient to infinite dilution is also given and marked with a star.

average size appears too large. Sizes obtained from XRD measurements do not represent the particle size but the size of the crystal domains within the particle. Since we do not necessarily have perfect single crystals the particles may consist of more than one domain, and the size would be potentially underestimated. The optical diameter is in the same size region as the crystallite size calculated from XRD patterns. Since the optical diameter is calculated based on an electronic transition, we conclude that it corresponds as well to the crystalline regions in the particle and not to the actual particle size measured with TEM, AUC, aF-FFF, and DLS. This hypothesis is strengthened by the exciton Bohr diameter which is $5 \mathrm{~nm}$ for $\mathrm{ZnS} .^{33}$ Methods that determine the actual particle size yield particle sizes above $5 \mathrm{~nm}$, which would correspond to an absorption onset at $340 \mathrm{~nm}$, which is not observed.

If we keep in mind that the investigated nanoparticles can be considered as core-shell nanoparticles consisting of a $\mathrm{ZnS}$ core and a cysteine shell, these observations point to an important difference between the different analytical methods. The particle size from TEM, XRD, and UV-vis absorption spectroscopy can at the best give the size of the crystalline particle core; in case of defects XRD and UV-vis absorption yield a smaller size of singlecrystalline domains, whereas the size from TEM can be bigger, if inner aggregate interfaces cannot be recognized. AUC, DLS, and aF-FFF on the other hand are all hydrodynamic techniques, which rely of transport processes of the particles in the solvent.

(33) Bhargava, R. N.; Gallagher, D.; Hong, X.; Nurmikko, A. Phys. Rev. Lett. 1994, 72, 416
Therefore, these techniques will determine the size of the nanoparticle including the shell and attached solvent. The layer thickness of similar stabilizer molecules on CdS was determined to be $0.4-0.5 \mathrm{~nm} .{ }^{34,35}$ This means that, for a nanoparticle with a $4 \mathrm{~nm}$ crystalline core (XRD, UV-vis), the hydrodynamic particle size should be $4.8-5 \mathrm{~nm}$. AUC is the only technique that roughly reproduces this average, whereas aF-FFF is affected by nanoparticle charge and DLS suffers from aggregates and polydispersity due to the lack of sample fractionation.

Figure 5 shows cumulative plots for the AUC, TEM, and DLS distributions. It is obvious that none of the distributions agrees with each other due to the reasons mentioned above. TEM suggests a broader distribution which might be attributed to the presence of agglomerates resulting from sample preparation. Figure 5 right also shows the effect of weighing. For TEM, the mass-weighted particle size distribution overestimates larger and aggregated particles and therefore suffers from aggregation problems of samples due to the drying step in sample preparation.

\section{CONCLUSION}

The determination of the particle size of nanoparticle dispersions is not as trivial as it may sound. There is a range of methods available, but the user must always keep in mind what the different techniques will detect if the nanoparticle is not a single phase but more complex like a core-shell particle as in the chosen example. For stabilized inorganic nanoparticles, hydrodynamic techniques will always detect the nanoparticles including the shell, whereas XRD or UV-vis spectroscopy is only sensitive to the crystalline core and XRD or UV-vis detects the size of defectfree domains rather than the particle size. This is valid for stabilizers not absorbing light or overlaying the spectrum of the nanoparticle, which could make the observation of the onset of absorption impossible for the nanoparticle. The particle size detected by TEM depends on the contrast of the usually organic shell. AUC-derived results have to be extrapolated to infinite dilution if concentration and charge effects need to be excluded. This requires measurement of a concentration series. However, this extrapolation is not practically possible for the whole distribution but can be done for the average particle size. If a distribution

(34) Börger, L.; Cölfen, H. Prog. Colloid Polym. Sci. 1999, 113, 23.

(35) Vossmeyer, T.; Katsikas, L.; Giersig, M.; Popovic, I. G.; Diesner, K.; Chemseddine, A.; Eychmüller, A.; Weller, H. J. Phys. Chem. 1994, 98, 7665. 
is required, it can only be measured at the highest possible dilution. However, the determined particle size will be too low in case of strong charge-charge interactions as in our sample, where $d_{\mathrm{H}}=4.5 \mathrm{~nm}$ was found for the highest measured dilution and $d_{\mathrm{H}}=4.9 \mathrm{~nm}$ from the sedimentation coefficient at infinite dilution for a density of $3.2 \mathrm{~g} / \mathrm{mL}$. This shows the necessity of the extrapolation for charged nanoparticles.

Also, the particle density in the dispersion has to be known for the calculation of the correct particle size because it can introduce a large inaccuracy. ${ }^{36}$ This is problematic in case of density distributions where the particle size distribution is folded with the density distribution. ${ }^{37} \mathrm{aF}-\mathrm{FFF}$ does not have this problem with density distributions since it detects the diffusion of the sample but suffers from unknown sample concentrations in the region of the accumulation wall, which can result in too small particle sizes for charged samples if no buffer can be applied to shield the charges.

DLS works well for very pure dispersions, which are hard to achieve. As the light scattering intensity is proportional to the sixth power of the particle radius, aggregates are problematic. We finally centrifuged the samples to minimize the noise, but it cannot be completely excluded that this alters the particle size distribution, although the elimination of particles smaller than 100 $\mathrm{nm}$ seems improbable, which is confirmed by the larger particle sizes from DLS compared to AUC and aF-FFF. Therefore, the problem of DLS was likely caused by the sample polydispersity.

The determination of particle size distributions by TEM is timeconsuming, and it has to be kept in mind that sample preparation is a very critical point. The main conclusion from our comparative investigation is that there is no "absolute" or "best" method of determining nanoparticle size (distributions) that is applicable to all situations. It is always desirable to apply several techniques

(36) Jamison, J. A.; Krueger, K. M.; Yavuz, C. T.; Mayo, J. T.; LeCrone, D.; Redden, J. J.; Colvin, V. L. ACS Nano 2008, 2/2, 311.

(37) Cölfen, H.; Völkel, A. Eur. Biophys. J. 2003, 32, 432. on the same sample like a hydrodynamic technique with one only sensitive to crystalline domains. For our example, combination of AUC and XRD or UV-vis spectroscopy can yield not only the size of the $\mathrm{ZnS}$ particle core but in addition the stabilizer shell thickness. Therefore, global analysis approaches which simultaneously take several analytical techniques into account for the evaluation are promising for the increasing complexity of nanoparticle systems. Nevertheless, it still has to be verified that the chosen technique does not suffer from artifacts like the too small particle diameter from aF-FFF and also AUC due to the particle charge or the uncertainty in TEM evaluations to recognize grain boundaries in aggregates. Therefore, it is clearly necessary to know about the strengths and weaknesses of the applied analytical techniques for nanoparticle size determination to recognize possible error sources. In addition, it has to be considered how the particle size (distribution) is weighted. It may therefore also be desirable to combine a technique like TEM, which yields number distributions and can detect small particles better, with a technique yielding a mass distribution, which weighs the larger particles more. We hope that our work could also contribute to an understanding of why there are often discrepancies between the results from different particle sizing techniques.

\section{ACKNOWLEDGMENT}

We thank the Swiss Commission for Technology and Innovation for their financial support of this work (CTI, TOP NANO 21 No. 4679.1). H.C. thanks the Max-Planck-Society for financial support. Antje Völkel (MPI) is acknowledged for the aF-FFF measurements.

\section{SUPPORTING INFORMATION AVAILABLE}

Additional information as noted in text. This material is available free of charge via the Internet at http://pubs.acs.org.

Received for review January 8, 2009. Accepted March 26, 2009.

AC900043Y 